\title{
Mejorando la calidad de los escritos: una experiencia pedagógica universitaria en el ámbito de la escritura en las disciplinas ${ }^{1}$
}

\author{
Improving the quality of writing: a pedagogical \\ university experience in the field of writing for \\ specific purposes
}

\author{
Mónica Tapia Ladino2 \\ Verónica Silva Madariaga 3
}

Resumen

En el ámbito universitario, la escritura de trabajos es una práctica común. El objetivo de este artículo es presentar una experiencia de intervención en el área de la escritura en las disciplinas. Se trabajó con una docente de Ingeniería comercial y una docente de Lengua Materna para mejorar la calidad de informes sobre una empresa realizados por estudiantes universitarios. Los estudiantes fueron organizados en grupos de tres, quienes elaboraron un escrito considerando la confección de dos borradores. El objeto de análisis corresponde a los borradores y el texto final escrito por 23 grupos. Los resultados indican que la docente logró mejorar la calidad de los escritos gracias a la incorporación de una metodología explícita para guiar el desarrollo de la tarea. Los trabajos mejor evaluados se caracterizaron por cumplir con los objetivos comunicativos correspondientes a la descripción de los recursos humanos de una empresa y proponer mejoras a partir del análisis de las debilidades.

\section{Palabras clave:}

Escritura en las disciplinas, didáctica universitaria, géneros discursivos.

\section{Abstract}

In the university context, writing is a common practice. The main aim of this paper is to present an intervention experience in the area of writing for specific purposes. This research was carried out with an academic of the Business Administration programme and one academic of the Spanish and Communication programme, with the aim of improving the university student reports quality about an enterprise. The students were organized in groups of three and presented 2 drafts before the final version of the report. The object of analysis corresponds to the drafts and the final report written by 23 groups. The results indicate that the business administration academic managed to improve the quality of the pieces of writing due to the introduction of an explicit methodology to guide the development of the task. The best evaluated assignments fulfilled the communicative objectives which corresponded to the description of the enterprise human resources and proposed improvements based on the analysis of its weaknesses.

Artículo recibido 15 de diciembre de 2009 y aprobado el 30 de abril de 2010.

1 Resultados obtenidos en el marco de la investigación denominada Escritura contextualizada en la Educación Superior: piloteo de dos programas de intervención para el desarrollo de habilidades escritas en el ámbito de las disciplinas. Proyecto C-14060/69 del Programa de Inicio de Carrera para jóvenes investigadores financiado por Fundación Andes.

2 Profesora de la Facultad de Educación de la Universidad Católica de la Santísima Concepción, Concepción, Chile.mtapia@ucsc.cl

3 Profesora de la Facultad de Ciencias Económicas y Administrativas de la Universidad Católica de la Santísima Concepción, Concepción, Chile. vsilva@ucsc.cl 


\section{Key words:}

writing for specific purposes, university didactics, gender discourse.

\section{Antecedentes}

En el desarrollo de una carrera universitaria es común exigir a los estudiantes la elaboración de textos escritos como una manera de evaluar su desempeño académico. Frecuentemente dichos escritos pretenden poner al estudiante en la tarea de realizar una actividad similar a aquella que desarrollarán en el mundo laboral real.

Para alcanzar sus objetivos pedagógicos, las carreras de pregrado desarrollan perfiles de ingreso y egreso con los que describen las conductas deseables para comenzar la formación y los resultados que esperan lograr al final del proceso. Esto quiere decir que si se considera deseable que un ingeniero comercial sea capaz, al momento de egresar, de "administrar empresas y organismos públicos y privados, utilizando los recursos para la creación de valor" 4 , entonces la universidad necesita proveer los ambientes y exigir las tareas que garanticen el desarrollo de tales capacidades. Una manera de visualizar el progreso, de una habilidad asociada a un perfil de egreso es vincular la habilidad con un producto escrito, por ejemplo, un texto del área profesional o disciplinar. Para lograr que los estudiantes elaboren textos escritos de calidad será necesario, por un lado, orientar la tarea de manera explícita y, por otro, asociarla al aprendizaje de las materias de formación.

Las experiencias en Chile sobre intervenciones pedagógicas para mejorar la calidad de los escritos en estudiantes universitarios han sido llevadas a cabo en el aula por académicos con formación en la enseñanza de lenguas (Sabaj, 2009., Neira, 2009). Estos trabajos han considerado el diseño y aplicación de un plan de intervención pedagógica que pone énfasis en el desarrollo de textos reales necesarios para enfrentar la vida académica, como el artículo científico o el informe de investigación. Se trata de experiencias hechas por actores que no pertene-

$4{ }^{1}$ Según los criterios de evaluación de la carrera Ingeniería Comercial de la Comisión Nacional de Acreditación. cen a la comunidad discursiva de los estudiantes en formación, sino de docentes del área de lengua materna que han apoyado y guiado el desarrollo de escritos en áreas disciplinares diferentes.

En el presente artículo compartimos una experiencia en la que una académica del área de Administración de Recursos Humanos intervino -durante tres cursos semestrales en 3 años- la calidad de los trabajos escritos de sus estudiantes. Tales informes daban cuenta del análisis de una pequeña empresa o Pyme con el fin de proponer innovaciones tendientes a mejorar su productividad. EL objetivo de esta actividad fue mejorar la calidad de los escritos de un informe en un curso de Recursos Humanos con el apoyo y orientación de la académica que dictaba dicha materia. Parar lograrlo, la docente, en un primer momento, fue capacitada en un enfoque de escritura como proceso y de escritura en las disciplinas, y en sucesivas instancias fue acompañada en el proceso de análisis de los logros. Esta investigación forma parte de un proyecto mayor de Fundación Andes C-14060/69 que buscaba averiguar sobre métodos eficientes para guiar la escritura universitaria por docentes de especialidad con el fin de pensar en capacitaciones a grupos mayores.

En este contexto, el propósito de este artículo es describir una experiencia cuyo objetivo fue utilizar la escritura tanto para aprender las materias propias de una carrera del área de administración, como para demostrar capacidades de emprender una tarea típica del ámbito profesional real, mediante el análisis y realización de textos escritos.

El supuesto de la investigación se basó en el movimiento de la escritura en las disciplinas (Bazerman, 2005) que señala que los modos de leer y escribir varían entre los diferentes ámbitos del saber, por lo tanto, requiere que los especialistas de dichas áreas se hagan cargo no solo de enseñar las materias, sino también de enseñar a leer y escribir sobre ellas.

El artículo contiene, en primer lugar, un marco referencial donde se revisan los sustentos teóricos provenientes del enfoque de escritura como proceso, la concepción de los géneros discursivos y la escritura en las disciplinas. En el apartado me- 
todológico, se presenta el diseño de investigación, en este caso, investigación-acción, y se describen los procesos realizados entre ellos la capacitación y la intervención. Luego los resultados se presentan organizados en cuatro grupos: el análisis de las notas de los informes realizados en 3 semestres, el análisis de la calidad general de los borradores realizados en cada caso, la descripción de los textos modelos y la valoración de la comunidad discursiva en este caso la docente responsable del curso.

\section{Marco teórico}

\section{Elecciones teóricas que fundamentan la intervención pedagógica}

La perspectiva de los modelos de escritura orientados hacia el proceso, se desarrollaron en el ámbito de la sicología cognitiva (Flower y Hayes, 1981., Flower, 1994 y Hayes, 1996). En el área de los procesos cognitivos que subyacen a la tarea de escritura se considera que están compuestos básicamente por tres fases: la planificación, textualización y revisión. Al estudiar escritores con diferente grado de destreza en la producción de textos escritos, Bereiter y Scardamalia (1987) avanzan hacia la explicación de la función epistémica de la escritura en sus modelos de "decir el conocimiento" y de "transformar el conocimiento". De este modo, los escritores menos avezados se circunscriben mucho más a realizar reproducciones poco elaboradas de aquello que desean comunicar, en cambio, los escritores aventajados suelen ajustar el texto a las condiciones retóricas en la que circulará el escrito. De este modo, los escritores avanzados transforman el contenido de sus escritos tanto para ajustarla al fin de la tarea como para hacerla comprensiva a los posibles lectores.

En una versión más contemporánea acerca de cómo se concibe el proceso de la escritura, se ha sumado la consideración de la variable sociocultural, es decir, el texto escrito como resultado de una interacción social particular. La producción y características de los textos dentro de comunidades discursivas es lo que algunos autores llaman géneros discursivos (Bajtin, 2002; Swales, 1990; Rastier, 2005), es decir, la institucionalización de actividades verbales que comparten todas aquellas personas que participan en determinados tipos de intercambios sociales y culturales por medio del uso de la lengua. Los géneros son consecuencia de la acción que se lleva a cabo con la palabra en respuesta a contextos sociales recurrentes. Cope y Kalantziz (1993) definen los géneros discursivos como "conventional structures which have evolved as pragmatic schemes for parking certain types of meaning and to achieve distinctive social goals, in specific settings, by particular linguistic means" (p.67). En este sentido cada área disciplinar decanta formas discursivas propias cuya inter comprensión es alta para quienes pertenecen a dicha tradición, sin embargo, para el estudiante que comienza a asomarse a ese campo, los textos resultan difíciles de comprender y aún más difíciles de producir (Tapia y Burdiles, 2005). Para Cope y Kalantziz (1993) la concepción del "género discursivo" es enormemente valiosa para la conceptualización de la relación profesor- estudiante. En ella, el alumno se concibe como un aprendiz que necesita conocer de manera explícita la forma de los textos y sus propósitos sociales y epistemológicos.

Dado los conceptos escogidos para elaborar esta investigación, aceptamos que el aprendizaje de la escritura no puede hacerse sino por la apropiación progresiva de las formas de uso, formas repetidas que llegan a ser rutinarias y se fijan como "maneras de decir". Resulta particularmente importante subrayar que la enseñanza de la escritura requiere de lo que Serafini (2000) llama un currículum progresivo que, al igual que la composición escrita, se caracteriza por avanzar en la consecución de tareas cada vez más complejas. Omitir esta señal, representa enseñar -como es frecuente observarlo en diversos contextos educativos- sin posibilidades de alcanzar progresos en los aprendizajes de la composición escrita, sino más bien provocando un resultado contrario: la incompetencia escritural y la desilusión del aprendiz ante el fracaso.

Frecuentemente en la formación universitaria, se desvincula el desarrollo de tareas, sean de producción de textos orales y escritos, de las temáticas de una clase. Muchas veces los docentes exigimos a nuestros estudiantes trabajos escritos cuyo proceso 
de elaboración desconocemos. En nuestra opinión, las tareas de escritura forman parte del proceso de aprendizaje de los contenidos de los programas de estudio. Así lo señala Carlino, (2005) al afirmar que "aprender los contenidos de cada materia consiste en una tarea doble: apropiarse de su sistema conceptual-metodológico y también de sus prácticas discursivas características, ya que una disciplina es un espacio discursivo y retórico como conceptual" (p 25).

Aunque se trata de tareas frecuentes, los estudiantes reciben escasa orientación y apoyo para elaborar trabajos escritos, como consecuencia, los profesores, muchas veces, no quedan conformes con la calidad de tales composiciones. Tal situación se debe al hecho de que los profesores sienten que dedicar tiempo de la clase para dicha actividad, los distrae de las actividades de la asignatura. Siguiendo a Carlino, opinamos que

\begin{abstract}
A pesar de que es cierto que en clase se exponen menos conceptos de la disciplina, sin duda los alumnos aprenden más contenido en su conjunto, porque la escritura aparece contemplada en el currículum y porque su inclusión contribuye a que los estudiantes se apropien de esas nociones expuestas por el docente, muchas de las cuales en ausencia de la escritura - habrían quedado sin poder ser incorporadas (Carlino, 2005, p. 53).
\end{abstract}

El tipo de escritura requerida en la universidad es de una especificidad particular, pues se realiza con fines muy particulares. Un movimiento que se ha preocupado de explicar este fenómeno con fines pedagógicos es la "Escritura a través del currículo" que se refiere específicamente a la atención pedagógica y curricular de la escritura que se desarrolla en las asignaturas universitarias como a los programas de composición que son ofrecidos desde los departamentos de lenguas. El movimiento ofrece estímulo sistemático, apoyo institucional y conocimiento educacional para aumentar la calidad de la escritura de asignaturas universitarias (Bazerman, et al., 2005, p. 9), porque entiende que se trata de un tipo de escritura al cual el estudiante universitario no había estado enfrentado antes.
Dentro del movimiento de Escritura a través del curriculum, se ha distinguido un área denominada "Escritura en las disciplinas" que realiza actividades referidas a la investigación para comprender qué ocurre realmente en las diferentes áreas disciplinares y se preocupa además de la reforma curricular para ofrecer instrucción de la escritura disciplinar, pero en un programa diseñado para tales propósitos (Bazerman, 2005, pp.9-10). La investigación sobre la Escritura en las disciplinas es un movimiento basado en la conciencia de los supuestos que tenemos sobre lo que constituye una buena escritura y cómo debiera ser enseñada, basada en los modelos de alfabetización. Asimismo, considera aquello que valora la comunidad discursiva como prácticas escriturales más adecuadas para la comunidad discursiva. En la idea de apoyar de manera inteligente la escritura a través del currículum, necesitamos conocer, entre otros temas, qué aspectos de la escritura se valoran y cuáles son los modos más fructíferos para lograr escritos de calidad más allá de la asignatura de lengua materna.

Los trabajos que los estudiantes universitarios escriben, forman parte de los géneros especializados dado que se trata de textos no cotidianos que se elaboran dentro de dominios conceptuales particulares y que revelan una función básicamente referencial, y cuyo contexto de circulación es una comunidad universitaria específica (Parodi, 2008). Dentro de los géneros especializados, reconocemos géneros académicos como aquellos que se elaboran preferentemente dentro del ámbito universitario. Como parte de su proceso de formación, los estudiantes universitarios requieren ingresar a la comunidad discursiva de su especialidad con el fin de mantener y extender el conocimiento de su área y así pasar a ser miembros del grupo. El discurso académico es epistémico y constitutivo del conocimiento del grupo (Herzberg, 1986, p.1).

Para poder ser parte de la comunidad discursiva, el estudiante novicio necesita acceder a los conocimientos mediante la lectura y la escritura. Para ello, se somete a una serie de actividades formales como la realización de cursos, la evaluación de sus aprendizajes mediante diversos sistemas y la interacción 
con su comunidad de profesores y compañeros. Por lo tanto, se trata de un proceso paulatino en el que adquiere y produce conocimientos especializados (Parodi, 2008).

Para el caso reportado en este artículo describimos el proceso de enseñanza aprendizaje de un tipo de texto que elaboran estudiantes universitarios en el ámbito académico universitario. Tales textos pretenden parecerse a un tipo de escrito que se requiere en el ámbito profesional, es decir, es un texto que se pide para desarrollar habilidades que se encuentran consignadas en un perfil de carrera para desarrollar una capacidad propia de un profesional de la Ingeniería Comercial. Por lo tanto, los textos que producen los estudiantes no corresponden a textos prototipos constituyentes de un género de especialidad, sino a textos que se producen en la comunidad académica, pero que se encuentran dentro del continuum entre el discurso académico universitario y el discurso profesional. Se trata, en este caso, de escritos que forman parte de lo que se denominan genéricamente 'informes académicos', los que se han entendido como "el conjunto de textos escritos por estudiantes en el ámbito académico para dar cuenta, de manera fidedigna, a un docente de un estado de conocimiento teórico y/o experimental, producto de la exploración de una realidad observada directamente o analizada desde la documentación” (Núñez y Espejo, 2005). Para el caso que estudiamos, el informe tiene una finalidad pedagógica que es "medir el dominio de una información o la de demostración de una habilidad para conocer la disciplina o emular situaciones de la vida profesional" (Tapia y Burdiles, 2009).

El objetivo de este trabajo es, por una parte, presentar una experiencia pedagógica universitaria orientada a mejorar la calidad de un escrito de carácter profesional con la guía de un docente especialista en la disciplina. Por otra parte, queremos demostrar que es posible mejorar una práctica pedagógica en el tiempo cuando se asocia una tarea de escritura típica de la profesión con el aprendizaje de una materia. En este caso, se trata de informes que dan cuenta de la evaluación crítica de empresas visitadas en terreno por estudiantes universitarios.

\section{Metodología}

El presente trabajo da cuenta de un estudio bajo el paradigma de la investigación-acción, dado que fue realizada por dos docentes que se interesaron en averiguar sobre el rol de la escritura en el proceso de enseñanza-aprendizaje (Mertler, 2006). Así se entiende por 'investigación-acción' a una averiguación sistemática conducida por docentes para conseguir información sobre cómo operan los estudiantes, cómo aprenden y cómo enseñan los docentes (Mertler, 2006, p. 2). Se adoptó una metodología de investigación cuanti-cualitativa, pues pretende explicar razones del comportamiento de los sujetos participantes analizando tanto datos numéricos como la calidad general de los escritos. Desde el punto de vista cuantitativo, se trata de un estudio de tipo observacional, longitudinal en su diseño temporal, debido a que se comparan resultados en distintos puntos del tiempo.

Los datos son primarios, puesto que se obtuvieron directamente. Desde una mirada cualitativa, se refiere a una investigación que explora los rasgos discursivos de un grupo de escritos universitarios para explicar su calidad considerando la valoración de la comunidad disciplinar.

\section{Los participantes}

Para la realización de este trabajo, participaron una académica responsable del curso Administración de Recursos Humanos dictado a Ingeniería Comercial en la Facultad de Ciencias Económicas y Administrativas de la Universidad Católica de la Santísima Concepción, y dos grupos de estudiantes que tomaron la asignatura de Recursos Humanos en el segundo semestre de los años 2006, 2007 y 2008.

Para informar esta experiencia hemos distinguido diferentes focos de análisis: la revisión de las prácticas originales llevadas a cabo por la docente antes de 2006, un período de capacitación para orientar la escritura con un enfoque de proceso y de escritura en la disciplina, y tres períodos de monitoreo de la experiencia. Cada inicio y fin de semestre de cada año, las docentes se reunieron para revisar los resultados y, a la luz de ellos, incorporaron mo- 
dificaciones para mejorar el logro de los aprendizajes de los estudiantes.

En todos períodos, la docente solicitó a sus estudiantes el desarrollo de un trabajo escrito. Para tal actividad, la académica recibió cada 15 días durante 4 meses del primer semestre del año 2006, una capacitación para apoyarla en la manera como tutelar los escritos de sus estudiantes. Dicha capacitación, la realizó una especialista en escritura universitaria del Departamento de Lenguas de la Facultad de Educación de la misma casa de estudios. La capacitación consistió, en primer término, en conocer el objetivo de la tarea de escritura que originalmente se desarrollaba para la asignatura Administración de Recursos Humanos. Conocida esa información, las académicas analizaron en conjunto los materiales reales de semestres anteriores con el fin de identificar los aspectos más relevantes que valora el ámbito profesional en esos tipos de textos. Luego de ello, la especialista asesoró en la elaboración de materiales para modelar la escritura y para crear indicadores que permitieran evaluar la confección de los textos mediante la revisión de borradores y el informe final.

Para mejorar la calidad de los escritos y, en consecuencia, la calidad de los aprendizajes, con la nueva metodología, la académica de la facultad de Economía organizó el curso en grupos de tres alumnos quienes debían elaborar su trabajo escrito final, considerando la confección de dos borradores. Para aplicar la metodología de proceso, la docente apoyó personalmente la elaboración de los borradores mediante tutorías que presentaron variaciones y elementos constantes entre la versión del año 2006 al 2008. Las variaciones consistieron en la obligatoriedad de las tutorías en el 2007 y 2008 para guiar la calidad y el avance de los borradores.

\section{Los escritos}

Los escritos realizados por los estudiantes tratan sobre la descripción de una pequeña empresa o Pyme. Con el objetivo de analizar la administración de sus recursos humanos. Para ello, los estudiantes realizaron varias visitas a la empresa, entrevistaron a los jefes y a los empleados y elaboraron un in- forme descriptivo y crítico con el fin de proponer un plan de intervención que permitiera mejorar la gestión de los recursos humanos en la empresa estudiada. Es importante resaltar que el texto escrito fue el resultado de la unión entre una fase descriptiva y otra analítica.

\section{El objeto de análisis}

El objeto de análisis de esta investigación corresponde al análisis de dos borradores y el texto final escrito por 23 grupos de 3 a 4 alumnos. De ellos, 8 trabajos corresponden a los realizados en el año 2006, 9 al año 2007 y 6 al 2008 en la carrera de Ingeniería Comercial. En total se trabajó con 69 escritos.

Para el análisis cuantitativo, se realizó la prueba estadística de varianza unidireccional para analizar si los promedios de las calificaciones obtenidas, en los tres trabajos escritos de cada año y los promedios de notas finales obtenidas por año, variaban significativamente. Asimismo, se aplicó la prueba $t$ para saber si los valores entre los promedios del primer borrador y el trabajo final presentaban diferencias significativas, así como calificaciones promedios del primer año y el tercero.

Para el análisis cualitativo, se consideró la revisión de la calidad de los aspectos textuales discursivos de los borradores y el escrito final por grupo, el análisis de las calificaciones de los borradores y la revisión de la calidad de la propuesta de intervención en la empresa. Para el análisis, se consideraron aquellos aspectos que la docente de la especialidad consideraba adecuados. Por lo tanto, se realizó un análisis de los textos mejor evaluados según el criterio de la docente que dictaba el curso y que, en definitiva, representa lo valorado, teniendo como criterio las competencias que los estudiantes debieran desplegar cuando se encuentren trabajando en el medio laboral.

\section{Resultados}

\subsection{Análisis de pautas de evaluación y calificaciones}

El primer grupo de resultados, se relaciona con el análisis de las notas de los informes realizados en los tres semestres de cada año. Antes de presentar 
tales datos, mostramos, de manera resumida, los criterios que sirvieron para elaborar las pautas utilizadas para evaluar y monitorear los dos borradores y el trabajo final.
En la Tabla 1, se presentan los aspectos que se valoraron en los informes de las empresas, mediante la aplicación de pautas y criterios para evaluar las tres fases de la escritura del texto.

Tabla 1. Aspectos valorados en cada uno de los escritos

\begin{tabular}{|l|l|l|}
\hline \multicolumn{1}{|c|}{ Borrador 1 } & \multicolumn{1}{c|}{ Borrador 2 } & \multicolumn{1}{c|}{ Trabajo Final } \\
\hline $\begin{array}{l}\text { Aspectos formales como } \\
\text { Índice } \\
\text { Jerarquización temática } \\
\text { Uso de lenguaje técnico }\end{array}$ & Aspectos formales & Aspectos formales \\
\cline { 3 - 3 } & Descripción de la empresa & Descripción de la empresa \\
\hline Descripción general de la empresa & $\begin{array}{l}\text { Propuesta de un plan de mejoramiento } \\
\text { en Recursos Humanos }\end{array}$ \\
\hline Fase descriptiva & Fase descriptiva & Fase descriptiva + Fase analítica \\
\hline
\end{tabular}

Al revisar la tabla, se advierte que el primer borrador tiene como énfasis los aspectos formales del diseño del texto, es decir, si bien evalúa la descripción de la empresa, valoraba más la presentación organizada de la información y la asunción de las convenciones de presentación de un texto académico. Para este caso, el borrador 1 dio cuenta del primer acercamiento de los estudiantes a la empresa, por lo tanto presentó una visión general de la visita en terreno. En la evaluación y monitoreo del borrador 2, se asignó menos peso a los aspectos formales del texto y se solicitó más información sobre la empresa. Para este caso, los estudiantes debían comentar aspectos como la historia de la empresa, la organización de los recursos humanos y su funcionamiento.

La versión final del trabajo fue evaluada, valorando con mayor énfasis la propuesta de mejoramiento de la empresa en lo relacionado con Recursos Humanos. Para esta fase de desarrollo del trabajo escrito, se solicitó un escrito más analítico basado en una buena descripción de la empresa. Por lo tanto cada borrador, guiado en tutorías por la docente, exigió más de parte del estudiante.

En el siguiente gráfico, se presentan los resultados de las calificaciones obtenidas por los estudiantes en el año 2006. Cada barra representa las fases del escrito: primer y segundo borrador, y trabajo final.

Gráfico 1. Calificaciones de informes finales de Recursos Humanos 2006.

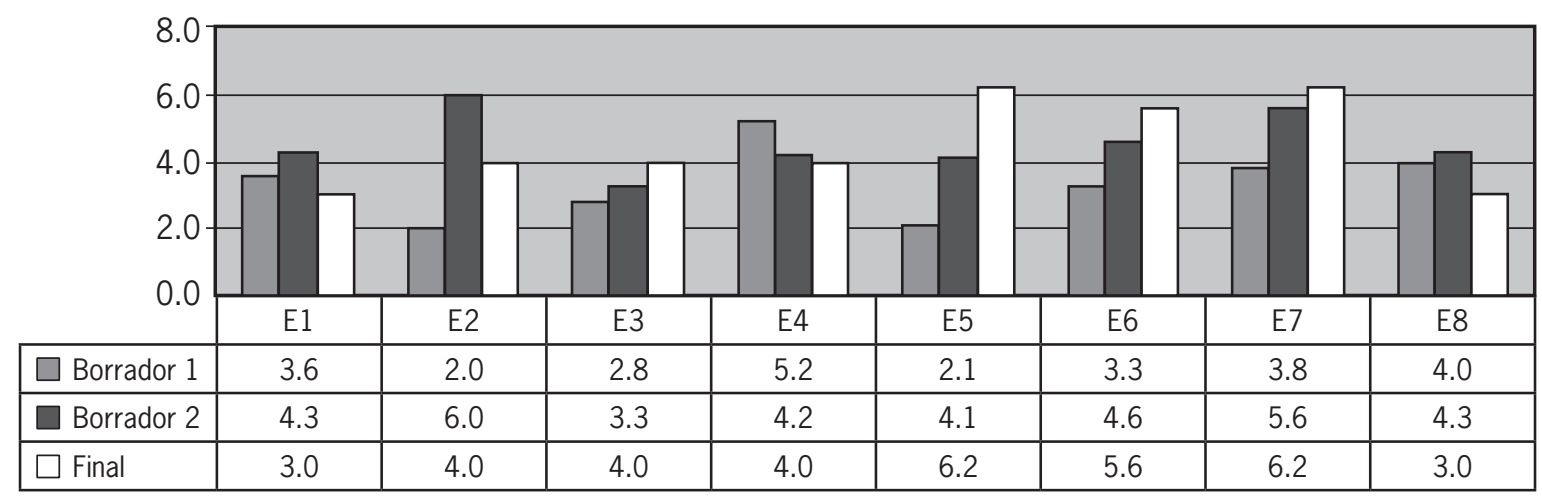

Calificaciones 
Como se observa en el gráfico 1, la mitad de los trabajos presentaron una baja en su evaluación hacía el tercer escrito. Es el caso de los escritos 1, 2, 4 y 8 . La otra mitad de los trabajos presenta una mejoría progresiva hacia el trabajo final, es decir, los estudiantes lograron subir sus notas durante el proceso de desarrollo del trabajo e informe. Se trata de los escritos 3, 5, 6 y 7 .

Al revisar las calificaciones, se aprecia que la mayoría de los grupos fueron calificados con notas bajas en su primer borrador (salvo el escrito 4). La tendencia muestra avances dispares, por un lado, pues se aprecia que cuatro trabajos mostraron una mejoría. Un trabajo comenzó con una buena evaluación, pero descendió durante el proceso. Otros mostraron una mejoría inicial, pero un descenso en la producción del texto final.
Cuando se aplicó el análisis de varianza unidireccional a los datos promedio entre el borrador $1,2 \mathrm{yel}$ trabajo final, la estadística indica que no hay diferencias significativas entre las tres medias, considerando un $5 \%$ de error. Dado que los valores promedios entre los escritos presentaron un alza (Borrador 1:3,3 y Trabajo final: 4.5), procedimos a aplicar la prueba $t$ que sí evidenció la presencia de diferencias significativas entre la calificación del primer borrador y el trabajo final $(\mathrm{t}[1,928]=14, \mathrm{p}: 0.695)$. Es decir, como la tarea de escritura se fue complejizando, la diferencia en los promedios de cada uno de los trabajos no presenta diferencias entre sí, pero la comparación entre el trabajo inicial y el final indica que los estudiantes, sí mejoraron la calidad de sus escritos.

En el gráfico 2 se presentan los resultados obtenidos en los borradores, y el escrito final en el año 2007.

Gráfico 2. Calificaciones de informes finales de Recursos Humanos 2006.

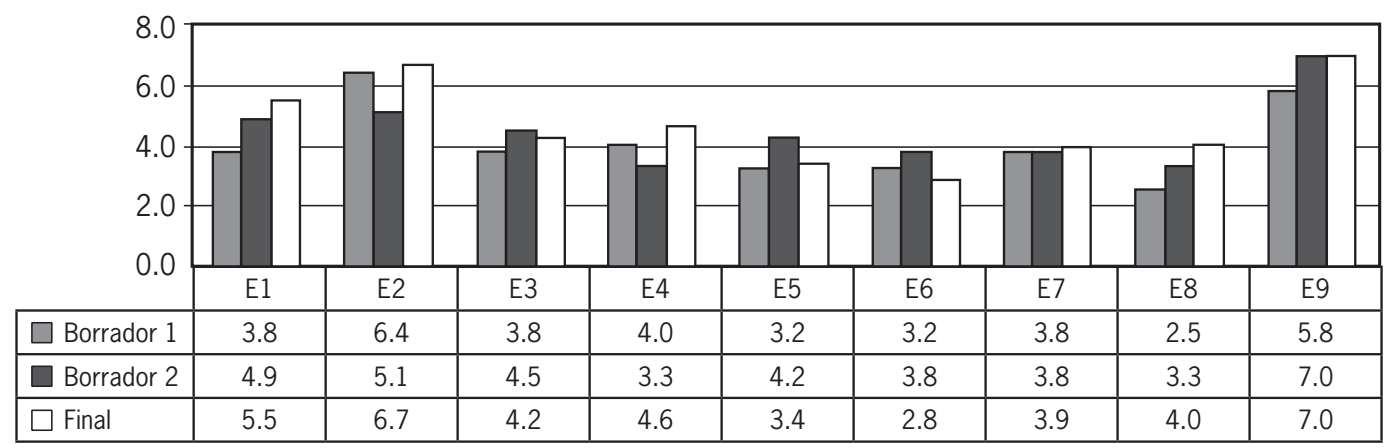

Calificaciones

Los resultados mostrados en el gráfico 2 indican que en seis de los nueve trabajos, se advierten mejoras durante el proceso de escritura (E1, E2, E4, E7, E8, E9). Solo en tres casos se advierte que los resultados descienden hacia el escrito final (E3, E5, E6). Llama la atención que todos los borradores $n .^{\circ} 1$ (salvo el E4) presentan notas reprobatorias. Sin embargo durante el desarrollo del trabajo escrito, se evidencia una mejora en la calidad hacia su versión final. Cabe destacar que el trabajo E3 si bien no asciende en la calificación del trabajo final, presenta un descenso leve.

En lo que respecta a las tendencias observadas de todo el grupo, 6 de los 9 mejoraron sus informes entre la primera entrega y la última, esto corres- ponde a un 66,6\%; lo que representa un ascenso en relación con el año 2006 donde el 50\% de los escritos mejora desde su etapa inicial (4 de 8 grupos).

Al igual que en el grupo 2006, el análisis estadístico de varianza unidireccional demuestra que no hay diferencias significativo entre las tres medias (Borrador 1: 4,1; Borrador 2: 4,4 y Trabajo final: 4,7). Sin embargo, al analizar estadísticamente las medias con la prueba $t$ del borrador 1 y el trabajo final, se advierte que hay diferencias significativas $(t[0,785]=10$, p: 0.7$)$. Es decir, la calidad de los escritos mejora progresivamente en el tiempo.

Un aspecto a destacar en esta segunda versión del curso son las altas calificaciones que obtuvie- 
ron algunos estudiantes, así: un 6,7 (E2) y un 7,0 (E9). Estos trabajos se caracterizaron por incluir propuestas de mejoras concretas a los presupuestos al interior de la empresa. Asimismo, el conjunto de propuestas se ajustaban al contexto de la empresa analizada, dado que se plantearon como viables y consensuadas con el empresario en terreno.

En el gráfico 2, se presentan los resultados de las calificaciones obtenidas por 6 grupos en el año 2008 .

Gráfico 3. Calificaciones de informes finales de Recursos Humanos-2008.

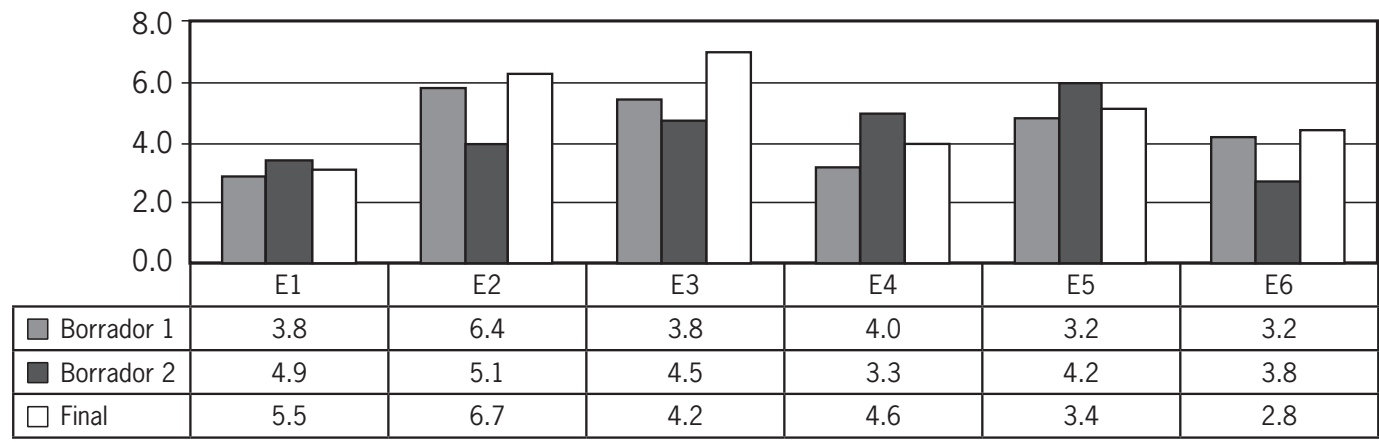

\section{Calificaciones}

El análisis de los datos del gráfico 3 indica que tres de los seis grupos (E2, E3, E6) mejoraron sus calificaciones desde el borrador 1 al trabajo final. Se destaca aquí, que las notas iniciales del borrador 1 , en general, son más altas que las mostradas en versiones anteriores, y las notas finales de aquellos trabajos que mostraron una mejora son más altas que en los años anteriores. Asimismo, llama la atención que en el borrador 2, los estudiantes presentan mayores dificultades, lo que se refleja en la disminución de la calificación, en alguno de los casos.

En relación con los análisis estadísticos, ocurre lo mismo a lo señalado en el año 2006 y 2007. No hay diferencias significativas entre las tres medias de las calificaciones (Borrador 1: 4,4; Borrador 2: 4,3 y Trabajo final: 5). Sin embargo al analizar, con la aplicación de la prueba $t$, las medias del borrador 1 y el trabajo final, se advierte que hay diferencias significativas $(t[0,87]=14$, p: 0.695$)$. Es decir, nuevamente durante la tercera intervención, mejora la calidad de los escritos de manera progresiva en el tiempo.

En el gráfico 4, se presentan los resultados de los trabajos escritos durante los tres años estudiados: 2007, 2008 y 2009 con el fin de presentar una visión que permita el establecimiento de comparaciones.

Gráfico 4. Comparación de calificaciones 2006, 2007 y 2008.

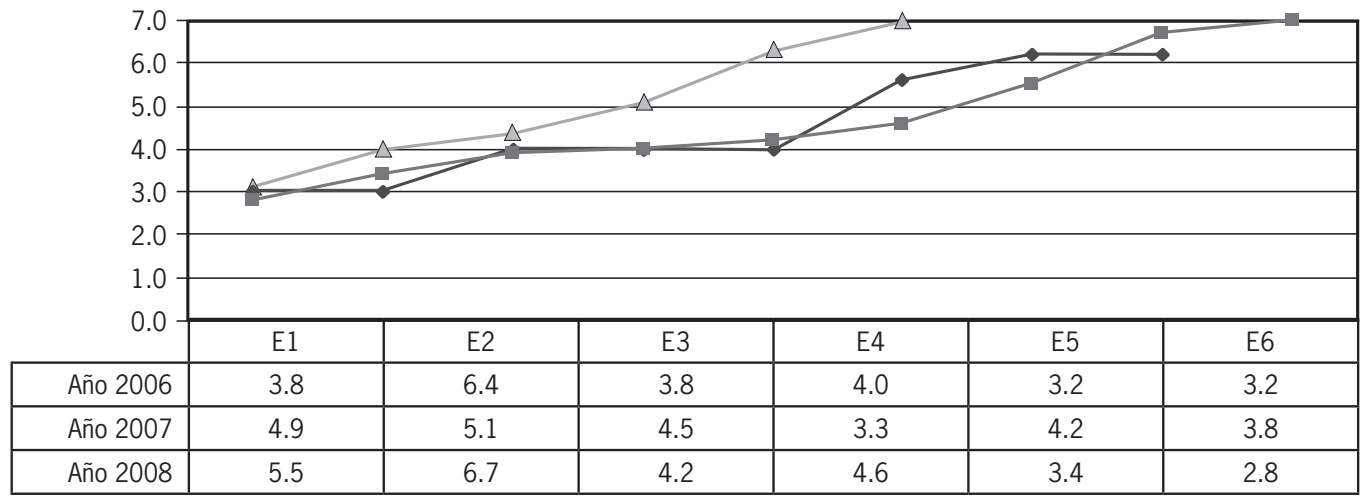

Calificaciones 
Al revisar la figura se aprecia que las calificaciones finales de los trabajos escritos durante los tres años estudiados han ido en ascenso. El punto inicial ha sido similar en cada año, pero el punto de llegada mejoró en los períodos 2007 y 2008 . Hay que agregar que esta mejoría posiblemente se debió al hecho de que las tutorías, en las cuales se revisaban los borradores fueron obligatorias durante dicho período.

La aplicación tanto de la estadística de la varianza unidireccional entre las medias de cada año como el análisis de la prueba $t$, en los promedios del año 2006 y 2008, demuestran que no hay diferencias significativas entre las medias.

En general se aprecia que las altas calificaciones, se relacionan con aquellos trabajos que presentan una completa descripción de la empresa, el texto se ajusta a la formalidad solicitada y es pertinente la propuesta de mejora de la empresa. En cambio, aquellos trabajos que presentan una calificación baja hacia el final, se caracterizan por presentar una adecuada descripción de la empresa, escasa formalidad en el texto, una baja calidad en el análisis de fortalezas y debilidades de la empresa, y una propuesta de mejoras inadecuada.

\subsection{Análisis textual discursivo de los trabajos modelos}

Para el análisis de los aspectos textual discursivo, se procedió a analizar 6 trabajos finales distribuidos en dos por año. Los trabajos escogidos presentaban las más altas calificaciones de cada grupo. En una primera instancia, se revisó y analizó cada trabajo completo y, en un segundo momento, seleccionamos el resumen ejecutivo del trabajo que obtuvo la calificación más alta. Para complementar el análisis, realizamos una breve entrevista a la académica de la especialidad quien con los 6 textos a la vista explicó las razones por las que cada trabajo obtuvo una alta calificación.

En relación con el análisis general de los textos presentamos la siguiente tabla que indica cuáles son las características y contenidos de cada una de las partes de los trabajos finales. Cada trabajo contaba con una portada, un índice que expresaba los contenidos jerarquizados y asociados a páginas, un resumen ejecutivo, la descripción pormenorizada de la empresa, un análisis crítico, la propuesta de mejora y anexos con materiales que apoyaban tanto la realización del trabajo (fotos) como los documentos de respaldo que describían las medidas a tomar para realizar las mejoras.

En la siguiente tabla, se presenta de manera resumida cada parte de los trabajos finales de Recursos Humanos de la carrera de Ingeniería Comercial.

Tabla 2. Descripción general de las partes del trabajo de Recursos Humanos.

\begin{tabular}{|c|c|c|c|c|c|}
\hline Índice & Resumen & Descripción del área RH & $\begin{array}{l}\text { Análisis } \\
\text { Crítico }\end{array}$ & Propuestas & Anexos \\
\hline $\begin{array}{l}\text { Identificación } \\
\text { de temas or- } \\
\text { ganizados je- } \\
\text { rárquicamente } \\
\text { y asociados a } \\
\text { páginas. }\end{array}$ & $\begin{array}{l}\text { Entre } 4 \text { a } 6 \text { párrafos } \\
\text { cada estudiante se hace } \\
\text { cargo de un tema, avan- } \\
\text { za desde la descripción, } \\
\text { el análisis de fortalezas y } \\
\text { debilidades de la empre- } \\
\text { sa hasta la elaboración } \\
\text { de una propuesta que } \\
\text { contenga soluciones. }\end{array}$ & $\begin{array}{l}\text { Punteo a modo de ficha con de- } \\
\text { talles de la empresa: historia, pla- } \\
\text { nificación estratégica, estructura } \\
\text { organizacional, descripción deta- } \\
\text { llada de los Recursos Humanos } \\
\text { (empleo, reclutamiento, selección, } \\
\text { contratación, inducción, capacita- } \\
\text { ción, sueldos, seguridad, higiene, } \\
\text { clientes y proveedores). }\end{array}$ & $\begin{array}{l}\text { Se identifican las } \\
\text { debilidades aso- } \\
\text { ciadas a los ítems } \\
\text { que describen la } \\
\text { empresa. } \\
\text { Cada crítica se } \\
\text { redacta de modo } \\
\text { independiente en } \\
\text { un párrafo. }\end{array}$ & $\begin{array}{l}\text { Cada propuesta se } \\
\text { redacta en párrafos } \\
\text { separados y cada } \\
\text { una incorpora ante- } \\
\text { cedentes sobre las } \\
\text { posibles soluciones } \\
\text { (cotizacones, datos, } \\
\text { entre otros). }\end{array}$ & $\begin{array}{l}\text { Fotos y } \\
\text { copias de } \\
\text { materiales } \\
\text { originales. }\end{array}$ \\
\hline
\end{tabular}

Como se aprecia en la Tabla 2, las partes del trabajo avanzan desde la presentación del trabajo, la descripción, el análisis de fortalezas y debilidades, y termina con una propuesta de mejoramiento que hace énfasis en la superación de debilidades mediante la adopción de cambios en la cultura empresarial.

Los escritos que se elaboraron, se caracterizaron, según lo planteado por la académica a cargo del cur- 
so, por la calidad de la descripción y el análisis de la empresa. Sobre el primer punto, la docente destacó la inclusión de detalles relevantes y no anecdóticos como la historia o evolución de la empresa, la identificación de información precisa sobre los diferentes aspectos de ella y la certeza de que lo escrito estaba redactado por los propios estudiantes. En el caso de algunos trabajos mal evaluados, la docente podía advertir qué partes de los escritos habían sido copiados de textos oficiales de la empresa y cuáles no.

En relación con el análisis de fortalezas y debilidades, la docente valoró la formulación de juicios críticos a partir de los datos, el diseño adecuado de un organigrama para aquellos casos en que la empresa no contaba con uno y, por lo tanto, fue necesario elaborarlo a partir de los datos obtenidos in situ. Sin duda, la formulación de un plan de mejoramiento adecuado sobre la empresa descrita fue el rasgo final que caracterizó los trabajos de más alta calificación. Esto quiere decir, que los estudiantes lograron proponer medidas ajustadas a las necesidades de la empresa, por ejemplo, en algunos casos se reportó que los funcionarios de la empresa necesitaban capacitarse en áreas que favorecían su labor, por ejemplo en temas como la productividad por un lado, y, por otro el grado de satisfacción del trabajador, favorecían su labor, por ejemplo, en temas como la productividad, por un lado y, por otro, el grado de satisfacción del trabajados, ya que estas capacitaciones incidían en su labor.

El análisis general de los aspectos comunes entre aquellos trabajos que recibieron mejores calificaciones, permite advertir que todos ellos son claros con el objetivo comunicativo de la tarea, es decir, elaborar un informe en que se analizara una pequeña empresa con el fin de sugerir mejoras en lo que a Recursos Humanos se refiere. Una parte que permite caracterizar de mejor forma este objetivo, es el análisis de la introducción del trabajo que recibió la mejor calificación.

En la Tabla 3, se presenta el análisis general de los objetivos comunicativos de cada uno de los párrafos correspondientes al resumen ejecutivo del trabajo mejor evaluado.

Figura1. Análisis de la estructura discursivo-comunicativa.

\section{RESUMEN EJECUTIVO}

El supermercado y panadería la Chilena se crea como una empresa familiar en el año 1940 por los padres del actual dueño don Andrés Salazar Alarcón. En sus comienzos la actividad económica radicaba en hacer pan para los vecinos en hornos de barro, luego comenzó a llegar la tecnología y con ellos mayores oportunidades de aumentar las ventas y la producción, hoy cuenta con 15 trabajadores y modernas maquinarias. Coelemu es uno de los mejores locales en su tipo. La empresa está constituida por su dueño, una cajera, dos contadores, panaderos, pasteleros, vendedoras y una persona encargada del aseo.

Con respecto al área de recursos humanos la empresa no cuenta con un departamento formal, pero sí realiza algunas actividades con relación al empleo de personal aunque sus métodos no sean del todo diferentes. La empresa intenta lograr que todos los puestos sean cubiertos por personal apto a través de reclutamiento por medio de avisos, de recomendaciones y de selección, analizando las habilidades de los empleados de contratación y haciendo un acuerdo con un contrato indefinido de inducción, dependiendo del caso de cada empleado.

Esta empresa no capacita a sus empleados, ya que considera que es un costo y no un beneficio, y con respecto a los sueldos todos reciben el sueldo mínimo excepto la cajera que recibe un sueldo más alto.

Los errores más recurrentes en este tipo de empresa son: asignación inadecuada de los costos, falta de capacitación a sus empleados y los escasos conocimientos técnicos de los dueños para dirigir la empresa, la falta de materiales necesarios para realizar la producción de los productos que comercializa la empresa y la inexistencia de un manual de funciones (descripciones de los cargos).

Para solucionar estas falencias proponemos algunas medidas que el gerente general debería considerar: capacitar al menos a los panaderos y pasteleros con un curso de pastelería básica en el cual podrán aprender a realizar una mayor variedad de productos, lo que aumentaría la diversidad de los productos ofrecidos en el local, cursos de administración para los dueños y realizar un conteo mensual de los materiales en bodega para prevenir la falta de utensilios, y también proponemos descripciones de cargo, las cuales servirán para encontrar personal con mayores conocimientos y habilidades de las que tienen los actuales trabajadores.

Presentación de la empresa: su historia e integrantes.

Descripción de los recursos humanos actuales.

Identificación de las debilidades sobre los Recursos Humanos.

Propuesta de soluciones para superar las debilidades. 
Como se aprecia en la figura, los estudiantes de ingeniería comercial que realizaron este trabajo describen la empresa, identifican con precisión los aspectos relacionados con el funcionamiento de los recursos humanos, presentan una propuesta de soluciones, todo de una manera concisa y ajustada a un tono de formalidad.

Como se aprecia en la tabla, cada párrafo contribuye al objetivo comunicativo general del escrito. Cada uno aporta nueva información y progresa temáticamente desde la descripción hacia el juicio analítico y al planteamiento de propuestas. Este breve análisis, no se propone como la estructura de un género o texto tipo, dado que en el corpus estudiado fueron muy pocos los estudiantes que alcanzaron la nota máxima. Es decir, no es nuestra intención declarar que la introducción antes presentada corresponda a la descripción del género resumen ejecutivo "Informe de recursos humanos", si no la descripción de los aspectos que un miembro experto de la comunidad valora como aceptable en un texto elaborado por un profesional en formación.

\section{Discusión}

La actividad de reflexión sobre la práctica docente permitió ir mejorando la calidad de los escritos. Por lo tanto, la adopción de una metodología de investigación-acción permitió optimizar la práctica docente y, al mismo tiempo, mejorar la calidad de los aprendizajes tanto en lo referido con el conocimiento de la asignatura como con la escritura de un texto propio de la profesión.

La aplicación de una metodología de proceso favoreció el desarrollo de habilidades de escritura al enfrentar la actividad. Esta modalidad demandó más actividad a la docente y más desafíos para los estudiantes, pues fue necesario monitorear el desarrollo de dos borradores y el trabajo final. La incorporación de cambios en la metodología de evaluación generó inicialmente resistencia en los estudiantes, hecho que se evidenció, en el primer semestre de 2006, en cuestiones como la inasistencia a las tutorías voluntarias, el incumplimiento en los compromisos de entrega de los avances y la dificultad para ajustar el escrito a los formatos solicitados.
Estos hechos, sin embargo, fueron disminuyendo en la medida que los estudiantes asistieron sistemáticamente a las tutorías que se volvieron obligatorias en el 2007 y 2008.

La aplicación de una metodología de proceso favoreció el desarrollo de habilidades de escritura y, por tanto, favoreció el desarrollo de textos que la comunidad de ingeniería comercial valora como adecuados para el proceso de formación. La escritura enfrentada como un proceso complejiza la tarea a los estudiantes, pues demanda de ellos más exigencias cognitivas, dado que para efectos del caso estudiado, los alumnos debieron obtener en terreno información de una empresa, organizar tal información para describirla, analizar sus fortalezas y debilidades, proponer modificaciones tendientes a mejorar la unidad de recursos humanos y, al mismo tiempo, ajustar las características de sus escritos a las exigencias del medio profesional.

En lo que se refiere a la evaluación de los textos escritos, la aplicación de pautas permitió observar que, en general, para los estudiantes parece más sencillo elaborar la primera etapa del trabajo que corresponde a la descripción de la empresa y al área de recursos humanos. En cambio, el análisis crítico, correspondiente al segundo borrador y al informe final, presenta mayores dificultades. Tal complejidad disminuye en la medida en que los estudiantes describen adecuadamente el objeto de análisis. De todos modos, desde el punto de vista estadístico se evidenció una mejora notable entre las calificaciones promedio entre el primer borrador y el trabajo final por año. El análisis de las tres notas no presentó diferencias estadísticas entre sí, lo que se puede explicar por qué cada versión exigía el desarrollo de una tarea más compleja. Por lo tanto, las diferencias más notables se dieron al comienzo y al final, lo que demuestra que las mejoras fueron progresivas y sostenidas.

Asimismo, en lo que se refiere al proceso de aprendizaje de la docente, ella reporta que conforme repetía y mejoraba su práctica a partir de la experiencia ganada año a año, el proceso de apoyo a los escritos se fue haciendo más llevadero, lo que le permitió obtener mejores resultados. Este mismo 
hecho puede explicar el hecho de que las diferencias entre las medias no fueran estadísticamente significativas entre sí. Esto quiere decir que la docente, cada año, fue reformulando su práctica y fue incorporando modificaciones para ir mejorando su intervención. Prueba de ello, se observa cómo en el tercer año de la intervención, la docente logra obtener calificaciones máximas que si bien no resultan significativamente estadísticas, cualitativamente sí son relevantes porque quiere decir que es posible acceder a un escrito ideal.

El análisis de uno de los mejores trabajos corresponde a la descripción de los aspectos que un miembro experto de la comunidad valora como aceptable en un texto elaborado por un profesional en formación. No pretendemos señalar que el trabajo corresponda a un género propiamente tal, sino a considerar la valoración de un miembro experto de la comunidad especializada como un actor que puede definir cuándo un texto es aceptable para su medio.

Sobre los aspectos discursivos que caracterizan a los trabajos mejor evaluados destacan los que se caracterizan por presentar una adecuada progresión temática evidenciada en el uso de estrategias que aseguraban la cohesión y la coherencia textual discursiva. En los trabajos se advierte un ajuste progresivo de los contenidos del texto en virtud de los objetivos comunicativos académicos para el cual fue solicitado. Es decir, los textos mejor evaluados están centrados en la función epistémica de la escritura, porque no solo logran describir la empresa, sino también realizan un análisis y proponen mejoras. Discursivamente se trata de un proceso de elaboración, reelaboración y ajuste progresivo del objetivo comunicativo.

Creemos que el acompañamiento de la elaboración de un trabajo escrito orientado al alcance de competencias laborales es una tarea que requiere un compromiso docente que se traduce, en este caso, en el conocimiento sobre proceso de escritura y una explicitación de las convenciones discursivas características de una comunidad disciplinar.

Hacerse cargo del aprendizaje de los estudiantes, aprovechando los recursos que nos ofrece la escritura permite mejores garantías de que las competencias que aspiramos alcanzar en los perfiles de egreso tengan más probabilidades de ser efectivamente logrados. Monitorear el logro de las diferentes habilidades que se requieren para la consecución de un perfil de egreso, mediante la producción de textos escritos, es hacer evidente el modo como se alcanzan diferentes niveles de formación. Creemos que una manera de evaluar el logro de los aprendizajes durante el desarrollo de una carrera es mediante la identificación de tareas de escritura y de lectura asociadas tanto al aprendizaje de las materias como al desarrollo de habilidades profesionales y académicas.

Lograr identificar dichas tareas permitiría pensar en el desarrollo de un currículo de escritura específico para el logro de capacidades profesionales. Una tarea como ésta involucra los esfuerzos de docentes de las disciplinas y de lengua materna.h 


\section{Bibliografía}

Bajtin, M. (2002). Estética de la creación verbal. Buenos Aires: Siglo veintiuno Editores.

Bazerman, C., Little, J., Bethel, L., Chavkin, T., Fouquette, D., Garufis, J. (2005). Reference guide to writing across the curriculum. Indiana: Parlor Press.

Beretier, C. y Scardamalia, M. (1987). The psichology of writen composition. New Jersey: Erlbaum.

Carlino, P. (2005). Escribir, leer y aprender en la Universidad. Buenos Aires: Fondo de Cultura Económica.

Cope, B. y Kalantzis, M. (1993). "How a genre approach to literacy can transform the way writing is taught". En Cope, B., Kalantzis, M. (Eds.). The powers of literacy: a genre approach to teaching writing. Pittsburgh: University of Pittsburgh Press.

Flower, L. y Hayes, J. (1981). "A cognitive process. Theory of writing". Collage Composition and Comunication, vol.32, pp.365-387.

Flower, L. y Hayes, J. (1996). "La teoría de la redacción como proceso cognitivo". En: Textos en contexto, pp.73-110. Buenos Aires: Asociación Internacional de Lectura.

Flower, L. (1994). The construction of negotiated meaning. A social cognitive theory of writing. Carbondale, IL: University of Southern Illinois Press.

Hayes, J. (1996). "A new framework for understanding cognition and affect in writing". En C. Levy \& S. Ransdell (Eds.). The sciencie of writing: theories, methods, individual differences and applications. Mahwah: Lawrence Erlbaum Associates.

Herzberg, B. (1986). “The politics of discourse communities”. Paper presentado en el CCC Convention. New Orleans. Marzo (en Swales 1990, p. 21).

Neira, A. (2009). "El género informe académico y su enseñanza a estudiantes universitarios: una propuesta metodológica”. Tesis para optar al grado de Magíster en Lingüística. Universidad de Concepción. Concepción, Chile.
Núñez, P. y Espejo, C. (2005). “Estudio exploratorio acerca de la conceptualización del informe escrito en el ámbito académico". En: Harvey A. (Comp.), En torno al discurso. Contribuciones de América Latina, pp. 135-148. Santiago: Ediciones Universidad Católica de Chile.

Parodi, G. (2008), (Ed.). “Géneros académicos y géneros profesionales: aproximaciones basadas en corpus". Valparaíso: Ediciones Universitarias de Valparaíso.

Rastier, F. (2005). "Situaciones de comunicación y tipología de los textos”. En: Habladurías, Universidad Autónoma de Cali. Facultad de Comunicación Social. Enero-junio. Año 2, n. ${ }^{\circ}$ 1, pp. 97-114.

Russell, D. (1990). "Writing across the currículo in historical perspective: toward a social interpretation". College English, vol.52, enero.

Sabaj, O. (2009). "Descubriendo algunos problemas en la redacción de Artículos de Investigación Científica (AIC) de alumnos de postgrado". Revista Signos, vol.42, n. ${ }^{\circ} 69$, pp.107-127.

Serafini, M. (2000). Cómo se redacta un tema. Didáctica de la escritura. Ciudad de México: Paidós.

Swales, J. M. (1990). Genre analysis: "english in academic and research settings. Cambridge: Cambridge University Press.

Tapia Ladino, M. y Burdiles, G. (2005). “Comparación de las demandas discursivas y cognitivas exigidas por el desarrollo de trabajos escritos de diferentes áreas disciplinares a estudiantes de pregrado de la UCSC". Ponencia presentada en el I Congreso Nacional de Estudios Comparados en Educación, organizado por la Sociedad Argentina de Estudios Comparados en Educación, 18 y 19 de noviembre.

Tapia Ladino, M. y Burdiles G. (2009). “Caracterización del género "informes" por los que se evalúa a estudiantes universitarios de la UCSC". En: Revista Letras. Universidad Pedagógica Experimental Libertador. Instituto Pedagógico de Caracas. 